\title{
Community-Acquired Acinetobacter baumannii Infections in Northern California
}

\section{Alex Studemeister*}

Division of Infectious Diseases, San Jose Medical Group, USA

\begin{abstract}
Acinetobacter baumannii is an emerging pathogen which causes serious healthcare-associated infections, and less commonly, community-acquired infections, especially in tropical and subtropical climates. A description of the epidemiological and clinical characteristics of patients with $A$. baumannii community-acquired infections, cared for a medical center located in Northern California, is presented. A total of 52 cases were identified, 11 (215) of which were community-acquired. Community-acquired $A$. baumannii infections caused a variety of serious clinical syndromes; most commonly wound infections, but also urinary tract infections, pneumonia and septic shock. Most cases were associated with comorbidities such as chronic obstructive pulmonary disease, diabetes mellitus and alcoholism. Although multi-drug resistance was seen in $20 \%$ of healthcar e-associated infections, all of the community-acquired infections were caused by fully susceptible strains.
\end{abstract}

Keywords: Acinetobacter baumannii; Community-acquired infections

Abbreviations: COPD: Chronic Obstructive Pulmonary Disease; UTI: Urinary tract Infection

\section{Introduction}

Acinetobacter baumannii is a gram-negative coccobacillus which has emerged as an important cause of healthcare associated infections, and wound infections in military veterans returning from Iraq and Afghanistan [1,2]. It may colonize tracheostomy sites, open wounds and environmental surfaces which serve as a nidus of spread via contaminated hands of healthcare personnel [3]. Hospital-acquired infections are characterized by high mortality rates and multi-drug resistance. Seasonal variation of A. baumannii infection rates has been noted with higher rates in the summer season [4].

Community-acquired A. baumannii infections have been recognized mainly in tropical and sub-tropical Asia-Pacific regions, such Taiwan, Hong Kong and Australia, and rarely in the United States [5]. Most reports describe patients with comorbidities, such as chronic obstructive pulmonary disease (COPD), renal failure and diabetes mellitus, although a few studies describe fulminant $A$. baumanii infections, such as pneumonia and severe sepsis, in otherwise healthy individuals. The mortality rate of community-acquired $A$. baumannii infections may be as high as $56 \%$. Most cases have been caused by strains susceptible to third generation cephalosporins and carbapenems. In order to expand on the epidemiology of communityacquired A. baumannii infections in the United States, a detailed report is presented describing clinical characteristics and outcomes of cases seen at a medical center in Northern California.

\section{Methods}

A retrospective study was conducted of patients diagnosed with A. baumannii infection in Regional Medical Center of San Jose, CA, a 247-bed trauma medical center serving Santa Clara County, from January, 2009, until July 2011. Patients with A. baumannii infection seen in the emergency department wound care clinic or within 48 hours of admission to the hospital, without a history of hospitalization or residence in a long-term care facility in the preceding 30 days, were compared to hospitalized patients with healthcare-associated $A$. baumannii infections during the same period. Patients were deemed to have A. baumannii infections if they fulfilled infection criteria according to the Centers for Disease Control and Prevention/National Healthcare Safety Network of the United States [6].

\section{Results}

A total of 52 cases of A. baumannii infections were identified (Table 1). Of these, 11 (21\%) were community-acquired, and consisted

\begin{tabular}{|l|c|c|}
\hline Variable & Community-acquired & Hospital-acquired \\
\hline Number (\%) & $10(20)$ & $41(80)$ \\
\hline Median age, years (range) & $50(27-91)$ & $73(40-96)$ \\
\hline Male sex & $8(73)$ & $30(73)$ \\
\hline Clinical syndromes & & \\
\hline Pneumonia (\%) & $2(20)$ & $25(61)$ \\
\hline Wound (\%) & $4(40)$ & $11(27)$ \\
\hline UTI (\%) & $3(30)$ & $5(12)$ \\
\hline Primary bacteremia (\%) & $1(10)$ & \\
\hline Associated conditions & & 10 \\
\hline COPD & 3 & 7 \\
\hline Diabetes mellitus & 1 & 8 \\
\hline Renal failure & 1 & 0 \\
\hline Homelessness & 2 & 0 \\
\hline Alcoholism & 3 & 15 \\
\hline Neurological & 1 & 2 \\
\hline Polytrauma & 0 & 0 \\
\hline Urethral stricture & 1 & 0 \\
\hline Cirrhosis & 1 & $32(4-81)$ \\
\hline Mean length of stay, days (range) & $7(3-8)$ & $4(10)$ \\
\hline Deaths (\%) & $2(18)$ & \\
\hline
\end{tabular}

Table 1: Clinical characteristics of $A$. baumannii infections, Regional Medica Center of San Jose, January 2009 to July 2011.

*Corresponding author: Alex Studemeister, Division of Infectious Diseases, San Jose Medical Group, Department of Medicine, Santa Clara Valley Medical Center, San Jose, CA, USA. Tel: 408-406-6282; E-mail: iddoc@earthlink.net

Received July 06, 2013; Accepted August 14, 2013; Published August 16, 2013 Citation: Studemeister A (2013) Community-Acquired Acinetobacter baumanni Infections in Northern California. Clin Microbial 2: 126. doi: 10.4172/23275073.1000126

Copyright: $\odot 2013$ Studemeister A. This is an open-access article distributed under the terms of the Creative Commons Attribution License, which permits unrestricted use, distribution, and reproduction in any medium, provided the original author and source are credited. 
Citation: Studemeister A (2013) Community-Acquired Acinetobacter baumannii Infections in Northern California. Clin Microbial 2: 126. doi: 10.4172/2327-5073.1000126

of 5 wound infections (45\%), 3 urinary tract infections (UTI, 27\%), 2 pneumonias (18\%), one of whom had bacteremia, and one bacteremia with shock (9\%). Both cases associated with bacteremia developed septic shock and died. Healthcare-associated infections (41 cases) included a predominance of pneumonia (59\%), and less commonly, wound infections (27\%), UTI (12\%) and bacteremia without death (2\%). Community-acquired infections occurred in younger patients (average age 54 years compared to 70 years). All community-acquired cases were caused by fully susceptible A.baumannii strains, whereas $20 \%$ of hospital-acquired infections were associated with multi-drug resistance to penicillins, cephalosporins and carbapenems. Most cases $(>75 \%)$ from both groups occurred during the months of December to May.

\section{Comment}

Community-acquired A. baumannii infections taken care of at a community-based medical center in Northern California, caused a variety of serious clinical syndromes, most commonly wound infections, but also UTI, pneumonia and septic shock (Table 1). In contrast, most healthcare-acquired cases were associated with pneumonia. Community-associated infections occurred in younger patients, and were lethal in both cases associated with bacteremia. Most patients in both groups had associated comorbidities such as COPD, diabetes mellitus and alcoholism. Unlike previously reported predominance in warmer months, most cases of both hospital and communityacquired A.baumannii infections were seen in late fall or winter months. Although multi-drug resistance was seen in $20 \%$ of healthcareassociated $A$. baumannii infections, all community-acquired infections were caused by fully susceptible strains. Local epidemiologic data, such as that found in this report, may be useful for the development of empiric treatment recommendations for A. baumannii infections by hospital-based antibiotic stewardship programs.

\section{References}

1. Fournier PE, Richet $\mathrm{H}$ (2006) The epidemiology and control of Acinetobacter baumannii in health care facilities. Clin Infect Dis 42: 692-699.

2. Centers for Disease Control and Prevention (CDC) (2004) Acinetobacter baumannii infections among patients at military medical facilities treating injured U.S. service members, 2002-2004. MMWR Morb Mortal Wkly Rep 53: 1063-1066.

3. Villegas MV, Hartstein Al (2003) Acinetobacter outbreaks, 1977-2000. Infect Control Hosp Epidemiol 24: 284-295.

4. McDonald LC, Banerjee SN, Jarvis WR (1999) Seasonal variation of Acinetobacter infections: 1987-1996. Nosocomial Infections Surveillance System. Clin Infect Dis 29: 1133-1137.

5. Falagas ME, Karveli EA, Kelesidis I, Kelesidis T (2007) Community-acquired Acinetobacter infections. Eur J Clin Microbiol Infect Dis 26: 857-868.

6. Horan TC, Andrus M, Dudeck MA (2008) CDC/NHSN surveillance definition of health care-associated infection and criteria for specific types of infections in the acute care setting. Am J Infect Control 36: 309-332. 